\title{
Disaster Recovery in Business Continuity Management
}

\author{
Jay S Patel' ${ }^{1}$, Keerthana V ${ }^{2}$ \\ 1MCA Student, ${ }^{2}$ Assistant Professor \\ 1,2Master of Computer Application in Storage and Cloud Technology, \\ ${ }^{1,2}$ Jain (Deemed-To-Be University) Bangalore, India
}

\begin{abstract}
How to cite this paper: Jay S Patel | Keerthana V "Disaster Recovery in Business Continuity Management" Published in International Journal of Trend in Scientific Research and Development (ijtsrd), ISSN: 24566470, Volume-3 | Issue-4, June 2019, pp.319-322, URL: https://www.ijtsrd. com/papers/ijtsrd2 3607.pdf

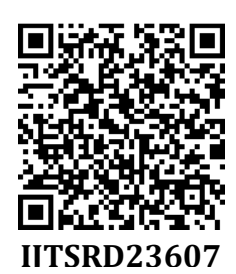

Copyright (C) 2019 by author(s) and International Journal of Trend in Scientific Research and Development Journal. This is an Open Access article distributed under the terms of the Creative Commons

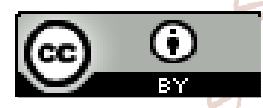
Attribution License (CC BY 4.0) (http://creativecommons.org/licenses/ by $/ 4.0$ )

There are many software, platform, and infrastructure servers in the cloud. Consumers are attached to the cloud server and can shop information over the internet and be admitted from anywhere to the statistics. It's a network of communication in real time. With the help of cloud access, we can run our programs from anywhere. We can access any software or statistics using the cloud without paying the cloud any money. Cloud computing is becoming increasingly popular in computing on a daily basis due to its ability to proportion globally distributed sources. Customers can access fully cloud-based services via the world's network right of entry. In the five continents, the largest IT companies are developing their information facilities to guide one of the kind cloud offers.[1]

Business continuity is vital requirements of many occupations as a sudden disruption of service can directly impact business goals causing significant losses in terms of receipts, reputation for business and market share losses. Therefore, the need for data recovery services is rising day by day and requires an efficient and effective data recovery technique to be developed. The recovery technique is intended to help the user collect data from any backup server when the server has lost its data and is unable to provide the user with data. Many organizations want to improve their ability to recover from system

Failures and loss of data, in particular to protect themselves against natural and man-made calamities.

\section{ABSTRACT}

Cloud computing is internet based computing technique where in systems are terconnected with sharing resources through every different. At present, every secure storage services. Data backup and Disaster Recovery Continuity issues are becoming fundamental in networks since the importance d social value of digital data is continuously increasing. Organization requires recovery requirements in terms of recovery time objective (RTO) and recovery point objective (RPO).Site recovery contributes to your business continuity and of azure VMs between regions, on-premises Virtual Machines and physical servers to azure, and on-premises machines to a secondary datacenter. The proposed system provides an extensive disaster recovery management using osoft Azure Recovery Vault Service. A back up is process on daily basis Which helps to Small And Medium-Sized Enterprises (SMEs) to cut down their

Keywords: Vacuole, The universe, dark matter, humans, structure

\section{INTRODUCTION}

Cloud computing is a completely computing technique based on the internet, in connect with sharing resources through each other.

A key undertaking in the presentation of DR services is to support Business Continuity (BC), allowing applications to return online quickly after a failure occurs.

A DR provider can also provide $\mathrm{BC}$, though commonly at excessive prices, by minimizing healing time there and the facts misplaced due to disaster. In this paper we explore how virtualized cloud platforms can be used to provide low-cost $\mathrm{DR}$ answers that we do not forget that in this work BC is a strict form of DR that calls for programs to renew full or partial operation shortly after a disaster.[2]

\section{LITERATURE SURVEY}

Various approaches and techniques have been conducted on cloud disaster recovery victimization. There are few works presented here, among them.

In [3], Wood et al proposed a new cloud service model, i.e. disaster recovery as a cloud service, to leverage cloud computing virtual platforms to prevent data disaster recovery. They created a disaster recovery cloud model for site applications that illustrated that high-cloud-based information backup can significantly reduce the company's data disaster recovery price. They did not, however, study the way to improve the quality of the service by using multiple clouds [4].

It has been demonstrated that the data center price includes the purchase of servers and infrastructure, the maintenance 
of facilities and the use of human resources. And there's no value difference that doesn't stand up to whether the service is standby or in use. Thus, if a service provider chooses to create a data center for disaster recovery on its own, it needs huge investment and results in tremendous waste generated by idleness resources attributable to the sporadic but imperative desire for disaster recovery.

The physical separation of the primary and backup sites is a key concept in a DRP. As shown in the table below, a significant fraction of disasters, including those caused by outages, are geographical.

\begin{tabular}{|l|c|}
\hline \multicolumn{1}{|c|}{ CAUSE } & ORGANIZATIONS \\
\hline System upgrades & $72 \%$ \\
\hline Power outage/ failure/ issues & $70 \%$ \\
\hline Fire & $69 \%$ \\
\hline Configuration management & $64 \%$ \\
\hline Cyber attacks & $63 \%$ \\
\hline Malicious employees & $63 \%$ \\
\hline Data leakage/ loss & $63 \%$ \\
\hline Flood & $48 \%$ \\
\hline Hurricane & $47 \%$ \\
\hline Earthquake & $46 \%$ \\
\hline Tornado & $46 \%$ \\
\hline Terrorism & $45 \%$ \\
\hline Tsunami & $44 \%$ \\
\hline Volcano & $42 \%$ \\
\hline War & $42 \%$ \\
\hline Others & $1 \%$ \\
\hline
\end{tabular}

The switch is called a failover when active processing of incoming transactions is switched from the failed primary to the backup site. After addressing the causes of the primary failure and returning the switch to the primary, the switch is called a failure. Depending on the nature of the backup site and how it links to the primary site process, a number of options arise. The situation of backup is often described as follows.

> Cold standby: recovery in such a case requires hardware operating system and installation of applications.

$>$ Hot standby: This requires a second data center that can provide availability in seconds or minutes. While the main site is down, a hot site can take over

$>$ processing. There may sometimes be a complete copy of the primary process in the backup, without installing either the OS or the application.

$>$ Warm standby: a hot and cold site tradeoff. Note that the terms "hot" and "warm" are defined differently at times.

\section{TRADITIONAL DISASTER RECOVERY}

It provides higher RPOs (Recovery Point Object) and RTOs (Recovery Time Object). Conventional geographic redundancy is an opportunity approach that has sufficient information facilities for shopping statistics while backup is being made. The installation of the same kind or hardware or software program to geo-redundant websites is essential to ensure speedy restoration time objective. Virtualization simplifies conventional disaster recovery by relaxing compatibility needs through the deployment of healing site hardware. Hardware configuration on the restore web page should be the same as the primary web page to hold the entire load of visitors served by the appropriate carrier on the affected web page holding full load of visitors served by impacted web page suitable carrier of high quality, reliability and latency. If programs are booted from scratch for disaster recovery, RTO should be comparable to RTO for conventional cold standby configurations on virtual machines.

A. Objective of recovery point: RPO is calculated for the maximum time taken for data loss when a disaster occurs. The vital RPO is commonly a commercial enterprise decision - really no records can be misplaced for a few programs $(\mathrm{RPO}=0)$, requiring non-stop synchronous replication to be used, while the suitable data loss may want to vary from a few seconds to hours or even days. The goal of the recovery point identifies how much information you are inclined to lose within a disaster event.

Your RPO is normally governed by the way you store and return information:

$>$ Weekly off-web page backups live on the absence of your mid-week statistics with statistical loss per week. Backups are even better every day off-web page.

$>$ Every day online backups on the website will continue to be lacking in your manufacturing environment with a day of statistical loss plus replicating transactions at some stage in the recovery period following the lack of the system.

B. Recovery time objective: RTO its miles of time that it can face up to and produce lower back to the machine when a disaster occurs. It might be minutes, hours, and days. It could also include detecting failure and getting the required servers ready to initialize an application that is interrupted in the execution center on the backup website.

The restore time goal identifies how in the event of a disaster a lot of downtime is acceptable.

Corporations and organizations can take advantage of DR offers that can be served by cloud service providers. Use of such offers, information security and carrier continuity are guaranteed exclusively for customers. In addition, a key issue in DR mechanisms is how cloud providers can tolerate disasters to prevent loss of statistics and disruption of service in their own information, infrastructure and services

\begin{tabular}{|c|l|c|c|}
\hline Tier & \multicolumn{1}{|c|}{ Description } & RTO & RPO \\
\hline 1 & Point time tape & $2-7$ days & $2-24 \mathrm{hrs}$. \\
\hline 2 & $\begin{array}{l}\text { Tape backup to } \\
\text { remote site }\end{array}$ & $1-3$ days & $2-24 \mathrm{hrs}$. \\
\hline 3 & $\begin{array}{l}\text { Disk point in time } \\
\text { copy }\end{array}$ & $2-24$ hrs. & $2-24 \mathrm{hrs}$. \\
\hline 4 & Remote logging & $12-24 \mathrm{hrs}$. & $5-30 \mathrm{~min}$ \\
\hline
\end{tabular}

\section{METHODOLOGY}

A vault for Recovery Services is a data-housing storage entity in Azure. The data is typically data copies or configuration information for VMs, workloads, servers, or workstations. For various Azure services such as IaaS VMs (Linux or Windows) and Azure SQL databases, you can use Recovery Services vaults to hold backup data. Vaults Recovery Services support DPM, Windows Server, Azure Backup Server, and more. The vaults of Recovery Services make organizing your backup data easy, while minimizing overhead management.[5] 
Recovery Services vaults are based on Azure's Azure Resource Manager model, whereas Azure Service Manager model was based on Backup vaults. When upgrading a Backup vault to a vault of Recovery Services, the backup data will remain intact during and after the upgrade process. Recovery Services vaults provide non-backup vaults features such as:

Improved capabilities to help secure backup data: Azure Backup provides security capabilities to protect cloud backups with vaults on Recovery Services. The security features ensure that your backups can be secured and data recovered safely, even when production and backup servers are compromised.

Central monitoring of your hybrid IT environment: you can monitor not only your Azure IaaS VMs, but also your onsite assets from a central portal with the vaults of Recovery Services.

Role-based access control (RBAC): RBAC provides control of Azure's fine-grained access management. Azure provides different built-in roles, and three built-in roles are available for Azure Backup to manage recovery points. The vaults of Recovery Services are compatible with RBAC, which restricts backup and restore access to the user roles set.

Protect all Azure Virtual Machines configurations: Vaults of Recovery Services protect resource manager-based VMs including Premium Disks, Managed Disks, and Encrypted VMs. You can upgrade your Service Manager-based VMs to Resource Manager-based VMs by upgrading a Backup vault to a Recovery Services vault. You can retain your VM recovery points based on the Service Manager while upgrading the vault and configure protection for upgraded (Resource Manager-enabled) VMs.

Instant restore for IaaS VMs: you can restore files and folders from an IaaS VM using the vaults of Recovery Services without restoring the entire VM, which allows faster restore times. For both Windows and Linux VMs, instant restore for IaaS VMs is available.[5]

Use Azure Backup MARS to Back Up Windows M achines Check the preconditions and create a vault for Recovery Services.

Download and set up the MARS Agent Build and schedule a backup policy.

\section{Regard to the MARS agent}

1. Azure Backup uses the MARS agent to back up files, folders and system status from on-site machines and Azure VMs to a backup vault in Azure for Recovery Services. You can run the agent as follows:

2. Run the agent directly on Windows machines on site so they can back up directly to Azure's backup vault for Recovery Services.

3. Run Windows-based Azure VMs (side-by-side with the Azure VM backup extension) to back up specific files and folders on the VM.

4. Run a Microsoft Azure Backup Server (MABS) or a Data Protection System Center-Manager (DPM) server. Machines and workloads return to MABS / DPM in this scenario, and then MABS / DPM uses the MARS agent to back up a vault in Azure. Depending on where the agent is installed, what you can back up.[6]

\section{DESIGN ARCHITECTURE}

Site recovery service: Site recovery helps keep business apps and workloads running during outages to ensure business continuity. Site Recovery replicates workloads from a primary site to a secondary location running on physical and virtual machines (VMs). When a primary site breakdown occurs, you fail to go to the secondary location and access apps from there. You may fail to return to it after the primary location is running again.

Backup service: By backing it up to Azure, the AZURE Backup service keeps your data safe and retrievable.

Availability set: Azure ensures that the VMs that you place in an Availability set run across multiple physical servers, compute racks, storage units, and switches to the network. If a hardware or software failure occurs, only a subset of your VMs will be affected and your overall solution will remain in operation. Sets of availability are critical to the development of reliable cloud solutions.

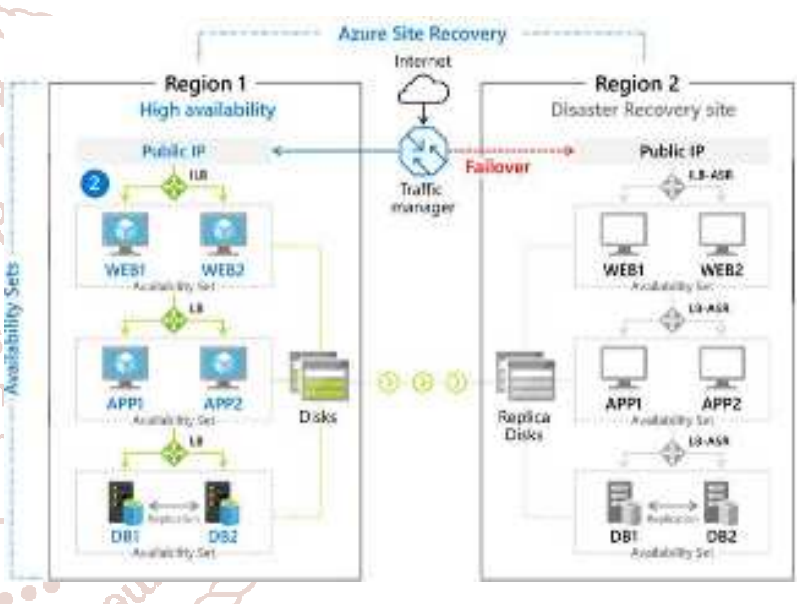

\section{CONCLUSION}

Traditionally, the importance of disaster recovery and business continuity has been to address and mitigate the cost of rebuilding the physical medium for service and delivery of products. However, with the information age and data digitization, a new medium-the information mediumhas now become a new frontier where disaster costs and potential impacts on business services also need to be addressed.

Fortunately, unlike the physical medium, it is not only possible to mitigate the information medium from the impact of disasters, but it can also be completely shielded from them by the methods of: data duplication, replication, and the use of internet data distribution. By using these methods, the information medium of an organization can be completely separated from the physical medium, resulting in cost mitigation and availability of service when the unforeseen occurs.

$>$ Understand the need for recovery from disasters

$>$ Analyze disaster impacts

$>$ Analyze business needs

$>$ Maintain executive and organizational cooperation

$>$ Make informed decisions on a suitable solution

$>$ Implement the solution and be ready 


\section{REFERENCE}

[1] A Study on cloud computing Disaster Recovery vol 1, issue 6, Aug 2013, IJIRCCE.

[2] T. Wood, E Cecchet, K. K. Ramakrishnan, P. Shenoy, J.van der Merwe, and A. Venkataramani, "Disaster recovery as a cloud service: economic benefits \& deployment challenges", Proc. 2nd USENIX Conference on Hot topics in cloud computing (HotCloud'10), Berkeley, CA, USA, 2010, pp. 8-8.

[3] Wood, Timothy, et al. "Disaster Recovery as a Cloud Service: Economic Benefits \& Deployment Challenges." Hot Cloud 10 (2010): 8-15.

[4] A. Greenberg, J. Hamilton, D. A. Maltz, and P. Patel, The cost of a cloud: Research problems in data center networks, ACM SIGCOMM Computer Communication Review, vol. 39, no. 1, pp. 68-73, 2008.

[5] https://docs.microsoft.com/enus/azure/backup/backu $\mathrm{p}$-azure-recovery-services-vault-overview

[6] docs.microsoft.com/en-in/azure/backup/backupconfigure-vault

[7] Lili Sun, Jianwei An, Yang Yang, Ming Zeng, "Recovery Strategies for Service Composition in Dynamic Network," International Conference on Cloud and Service Computing, 2011.

[8] Lenk, Alexander."Cloud Standby Deployment: A ModelDriven Deployment Method for Disaster Recovery 